\title{
ANALISI PERIODALE \\ APPLICATA AI MICROBAROGRAMMI
}

\author{
F. MosetTi
}

1. - Premessa. - In base ad una ricca casistica abbiamo potuto vedere $\left({ }^{2}\right)$ che esistono dei legami fra la presenza di aree cicloniche o di linee frontali (almeno entro una certa distanza dalla stazione registratrice) e il tipo di microbarogrammi registrati. Tali relazioni non vanno ricercate nelle onde microbariche a breve periodo (fino a 5 minuti circa) che, in base a quanto abbiamo potuto vedere, risultano dovute prevalentemente al vento, bensì nelle onde a lungo periodo (tra 10 minuti e 2 ore circa). Tra queste oscillazioni a lungo periodo va però fatta una distinzione essenziale a seconda che siano in rapporto coi fronti o coi cicloni. Un fronte $(2,5)$ dà al microbarografo corti gruppi di oscillazioni a lungo periodo, molto ampie e a carattere visibilmente smorzato, alle quali si sovrappongono oscillazioni pure ampie ma a periodo brevissimo che danno un'immagine vistosa e caratteristica della perturbazione in transito sulla stazione. Tali oscillazioni " da fronte " iniziano, a seconda dell'energia insita nella discontinuità, anche parecchie ore prima del passaggio di questa sulla stazione e svaniscono più o meno lentamente; la loro osservazione in reti di stazioni potrebbe essere di indubbia utilità, accanto agli usuali metodi, per il tracciamento della linea frontale e per seguirne $\mathrm{i}$ movimenti. Di aspetto completamente diverso sono invece le registrazioni di oscillazioni in rapporto con aree cicloniche. Tali onde sono praticamente sempre presenti, con maggior o minor ampiezza e con differenti periodi, su qualunque microbarogramma: esse formano una specie di substrato oscillante sul quale si evolvono le altre fluttuazioni meno persistenti quali quelle a loreve periodo in rapporto al vento, o quelle a lungo periodo legate al passaggio dei fronti. Considerando, specialmente in giornate estive, i microbarogrammi ottenuti in presenza di una sola area ciclonica $e$ in assenza di ogni altra apparente causa perturbatrice entro un raggio molto vasto, abbiamo potuto constatare che essi erano costituiti praticamente da un'unica periodicità il cui periodo cresceva col crescere 
della distanza tra la perturbazione e la stazione registratrice e la cui ampiezza era tanto maggiore, a parità di distanza, quanto maggiore era il gradiente barico dell'area ciclonica stessa. La presenza simultanea di più perturbazioni provocava interferenze nella registrazione, che diventava irregolare e tormentata.

Alcuni anni fa, all'inizio di questo nostro gruppo di ricerche, facevamo uso di uno strumento costruito dal Padre Alfani, molto sensibile ma con costanti valvolari ${ }^{(1)}$ e scorrimento del chimografo tali da essere utilizzabile solo per la registrazione di onde brevi (da qualche frazione di secondo a 1-2 minuti).

In seguito abbiamo fatto uso di strumenti con costanti valvolari idonee alla ricezione di onde a lungo periodo (da 2 minuti a 2 ore circa), con registratore a scorrimento più lento $(2 \mathrm{~cm}$ all'ora), e muniti di registrazione fotografica per eliminare il troppo incostante attrito del pennino sul foglio affumicato.

Il microbarografo, come si sa, è costituito essenzialmente da una capsula pneumatica $\left({ }^{1}\right)$ o da una lamina di gomma, tesa sull'apertura di un tubo, in comunicazione con un grosso recipiente (tanto più capace quanto più si vuol ingrandire l'escursione della pressione), che viene ad oscillare sotto la spinta delle fluttuazioni bariche. Poiché in tal maniera lo spostamento della capsula pneumatica o della lamina di gomma, prescindendo dalla risonanza dell'oscillatore meccanico, sarebbe eguale per qualunque periodo dell'onda di pressione da registrare, il recipiente viene messo in comunicazione con l'esterno attraverso un capillare regolabile, onde smorzare le oscillazioni a periodo più lungo.

Abbiamo infatti mostrato in $\left({ }^{1}\right)$ che la variazione del flusso oscillante $q$ con frequenza $\omega$ e ampiezza $p_{0}$ attraverso il capillare con cui il recipiente di sensibilità comunica con l'esterno è

$$
\frac{d q}{d t}=\frac{M \omega}{\sqrt{\omega^{2}+A^{2}}} p_{\mathrm{o}} \sin (\omega t+\varphi)
$$

ove $M$ ed $A$ indicano particolari costanti.

Se $\triangle p$ indica la differenza tra pressione interna al recipiènte $\mathrm{e}$ pressione atmosferica esterna, potremo anche scrivere, per la legge di Poiseuille:

$$
\frac{d q}{d t}=M \triangle p
$$


Dal confronto tra queste due espressioni si ottiene

$$
\triangle p=\frac{\omega}{\sqrt{\omega^{2}+A^{2}}} p_{\circ} \sin (\omega t+\varphi)
$$

Lo spostamento della capsula viene così ad essere proporzionale a $p_{o}$ sicché, a meno di una costante, e trascurando l'effetto dell'oscillatore meccanico, l'ingrandimento dinamico del microbarografo risulta variabile, in funzione della frequenza dell'onda da registrare, secondo la:

$$
S=\frac{\omega}{\sqrt{\omega^{2}+A^{2}}}
$$

Poiché la costante $A$ cresce con l'apertura del capillare, la funzione [1] è la legge dello smorzamento delle onde lunghe nel microbarogramına. Il corrispondente filtro è costituito dalla capacità del recipiente di sensibilità e dalla resistenza opposta dal capillare al passaggio dell'aria fra recipiente ed esterno.

Volendo smorzare anche le onde brevi col medesimo sistema, potremo ( ${ }^{4}$ ) racchiudere anche la capsula registratrice in un recipiente munito di capillare; in tal maniera, indicando con $B$ un'altra costante avente le medesime dimensioni fisiche $\operatorname{di} A$, si ricava facilmente ( ${ }^{\dagger}$ ) che l'ampiezza della registrazione varia proporzionalmente a:

$$
S^{\prime \prime}=\frac{\omega B}{\sqrt{\left(\omega^{2}+A^{2}\right)\left(\omega^{2}+B^{2}\right)}}
$$

Questa funzione si annulla sia per i piccoli periodi che per i grandi ed esalta la frequenza $\omega=\sqrt{A B}$.

Il recipiente di sensibilità del microbarografo, comunicante con l'esterno attraverso il capillare, funge dunque da filtro per le onde lunghe, mentre la scatola racchiudente la capsula registratrice, comunicante con l'aria esterna pure mediante un capillare, rappresenta il filtro per le onde brevi.

Pur avendo, l'onda selezionata, la frequenza $\omega=\sqrt{A B}$, spesso la selettività ad essa corrispondente non è sufficiente. Si può allora aumentare, da un lato o da entrambi, la riduzione d'ampiezza per $i$ periodi attorno a quello da selezionare, aggiungendo, ai due capillari preesistenti, un numero, eguale o no, di altri sistemi “ scatola di capacità + capillare $)$. In tal maniera, $B$ ' indicando la costante (eguale) di ogni coppia di filtri applicati alla capsula registratrice e $A^{\prime}$ indi- 
cando la costante delle coppie dei filtri applicati al recipiente, se $n$ e $m$ rappresentano il numero delle coppie, la sensibilità dello strumento viene rappresentata $\left({ }^{(}\right)$dalla

$$
S=\frac{\omega}{\sqrt{A^{2}+\omega^{2}}} \frac{B}{\sqrt{\omega^{2}+B^{2}}}\left(\frac{B^{\prime}}{\sqrt{\omega^{2}+B^{\prime 2}}}\right)^{\mathbf{0}}\left(\frac{\omega}{\sqrt{\omega^{2}+A^{\prime 2}}}\right)^{\mathrm{m}}
$$

Operando in tal modo, si ottiene, nel caso praticamente più semplice che $A=A^{\prime}=B^{\prime}=B$ e $n=m=p$,

$$
S=\left(\frac{\omega B}{\omega^{2}+B^{2}}\right)^{P}
$$

Con $p$ filtri, cioè, un'onda di pressione $P(t)$ composta, viene ridotta nella seguente maniera:

$$
\begin{gathered}
P(t)=\sum_{1}^{\Sigma_{\mathrm{i}}} a_{\mathrm{i}} \sin \left(\omega_{\mathrm{i}} t+\varphi_{\mathrm{i}}\right)-\sum_{1} \sum_{\mathrm{i}}^{\mathrm{K}}\left(\frac{\omega_{\mathrm{i}} B}{\omega_{\mathrm{i}}^{2}+B^{2}}\right)^{\mathrm{P}} a_{\mathrm{i}} \sin \left(\omega_{\mathrm{i}} t+\varphi_{\mathrm{i}}\right)= \\
=\frac{a_{\mathrm{r}}}{2 p} \sin \left(\omega_{\mathrm{r}} t+\varphi_{\mathrm{r}}\right)+\text { termini trascurabili }
\end{gathered}
$$

qualora si operi con filtri tali che $B=\omega_{r}$.

Ci siamo posti alla costruzione di un nuovo apparecchio di tal fatta per poter studiare, senza tema di incorrere in errori di interpretazione o di analisi, il comportamento e la correlazione di ogni tipo di onde registrabili coi microbarografi e soprattutto per tentare di definire, in maniera esauriente, la questione della provenienza delle onde microbariche.

Il microbarografo, munito dei due sistemi di filtri, può essere di utile applicazione anche quando non si voglia registrare proprio una sola delle periodicità presenti. Così il capillare (con o senza aggiunta di ulteriori filtri del recipiente di sensibilità) serve ad eliminare il contributo delle lunghe fluttuazioni (per es. variazione diurna della pressione atmosferica, di per se stessa molto ampia); tali fluttuazioni maschererebbero le oscillazioni più brevi e più interessanti, riducendo le funzioni di un microbarografo, strumento differenziale, a quelle di un comune barografo enormemente amplificato.

Racchiudendo invece la capsula registratrice in una scatola munita di capillare potremo eliminare le onde brevisssime (p. es. quelle dovute al vento), sovente molto ampie, che pure possono essere di noia in particolari ricerche. 
Nella fig. 1 portiamo un esempio di questa possibilità d'impiego; il grafico superiore rappresenta una registrazione microbarografica ottenuta con vento da ENE a $80 \mathrm{~km} / \mathrm{h}$ di velocità, con capsula registratrice in diretta comunicazione con l'esterno e capillare del recipiente di sensibilità abbastanza chiuso.

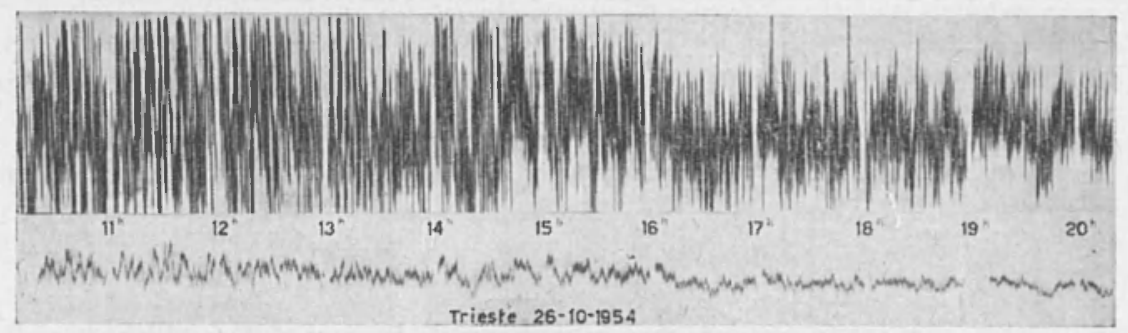

Fig. 1

Le onde più lunghe, con 3 o più ore di periodo, appaiono, dal grafico superiore, ricoperte dalle brevi e fortissime oscillazioni da vento che mascherano completamente le periodicità intermedie.

Il grafico inferiore è stato tracciato simultaneamente da uno strumento eguale al precedente ma fornito di filtri per le onde brevi: il

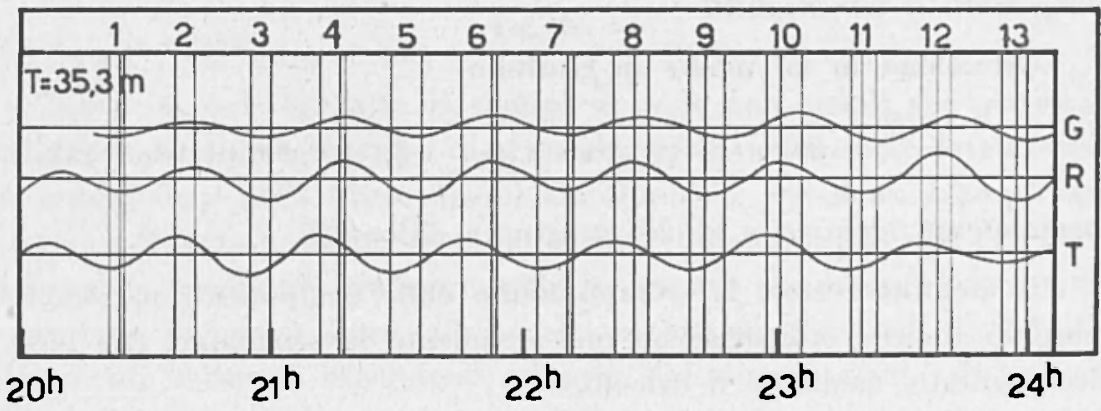

Fig. 2-a

contributo delle oscillazioni da vento è ridotto a piccole frangie che non impediscono di apprezzare la struttura e il tipo del "microba. rogramma residuo ").

2. - Analisi periodale delle onde microbariche. - Nella nota di cui al numero $\left({ }^{3}\right)$ della bibliografia auspicammo che si potessero conoscere con sicurezza il periodo e gli sfasamenti fra le varie onde, allo scopo di determinarne, senza ombra di dubbi, la direzione di prove- 
nienza e la velocità. Conseguenza logica di tutto questo, la costruzione del nuovo microbarografo.

Abbiamo tuttavia voluto vedere, in questo lavoro, quali risultati si ottengano quando si cerchi di calcolare la velocità di fase e la direzione di provenienza degli impulsi considerando oscillazioni ridotte ad un'unica periodicità, non grazie a particolari strumenti, bensi mediante l'applicazione di particolari operatori matematici all'oscillazione complessiva del microbarogramma. Abhiamo cioè sottoposto alcuni microharogrammi normali ad analisi periodale $\left({ }^{6}\right)$.

Con tale metodo, partendo dalla registrazione originaria del tipo

$$
P(t)=\sum_{\mathrm{i}}^{\mathrm{K}} a_{\mathrm{i}} \sin \left(\omega_{\mathrm{i}} t+\varphi_{\mathrm{i}}\right)
$$

si ottiene, mediante opportune combinazioni lineari delle ordinate del microbarogramma, una funzione

$$
\underset{\mathrm{i}}{\sum_{\mathrm{i}}^{\mathrm{K}}} M_{\mathrm{i}} a_{\mathrm{i}} \sin \left(\omega_{\mathrm{i}} t+\varphi_{\mathrm{i}}\right)
$$

ove $\quad M_{\mathrm{i}}=\gamma_{\mathrm{o}}+\gamma_{1} \cos \omega_{\mathrm{i}}+\gamma_{2} \cos 2 \omega_{\mathrm{i}}+\gamma_{3} \cos 3 \omega_{\mathrm{i}}+\ldots$

con $\gamma_{0}, \gamma_{1}, \gamma_{2} \ldots \ldots$ costanti da determinare.

Si possono calcolare gli $M_{\mathrm{i}}$ in modo che $M_{\mathrm{r}}=1$ e gli altri $M_{\mathrm{i} \neq \mathrm{r}}$ siano nulli o trascurabili.

Otteniamo in tal modo un risultato

$$
I^{\prime}(t)=\Sigma_{\mathrm{i}}^{\mathrm{K}} a_{\mathrm{i}} s i n\left(\omega_{\mathrm{i}} t+\varphi_{\mathrm{i}}\right) \rightarrow a_{\mathrm{r}} s n\left(\omega_{\mathrm{r}} t+\varphi_{\mathrm{r}}\right)+\text { termini trascurabili, }
$$

formalmente identico a quello raggiunto dalla $\left[{ }^{4}\right]$.

In definitiva cioè, la scomposizione nelle componenti periodiche semplici anziché ottenerla con uno strumento la otteniamo, con identico risultato, mediante il calcolo.

Abbiamo voluto sottoporre ad analisi periodale i medesimi diagrammi (giorno 27.8 .52 dalle $20^{\text {h }}$ alle $24^{\text {h }}$ e 29.8 .52 dalle $0^{\text {ht }}$ alle $5^{\text {h }}$ ) presentati nel lavoro di cui al numero $\left({ }^{3}\right)$ della bibliografia, ottenendo pressoché i medesimi risultati.

Il problema è interessante, nelle sue conseguenze, anche dal punto di vista della perfetta applicabilità del metodo dell'analisi periodale a diagrammi strumentali. Dai risultati di questa analisi abbiamo eliminato le onde di periodo più breve (di 2 e 3 unità di ascissa - l'unità di ascissa adottata è l'intervallo di 6 minuti) perché troppo influen- 
zate dalle irregolarità e dagli errori di lettura dei microbarogrammi e abbiamo portato invece la nostra attenzione sulle componenti pii lunghe; $i$ risultati di queste analisi sono raffigurate nelle figg. $2 a$ e $2 b$ e nelle figg. $3 a, 3 b$ e $3 c$ in cui si fa la comparazione tra le fasi nelle tre stazioni $G, R, T$.

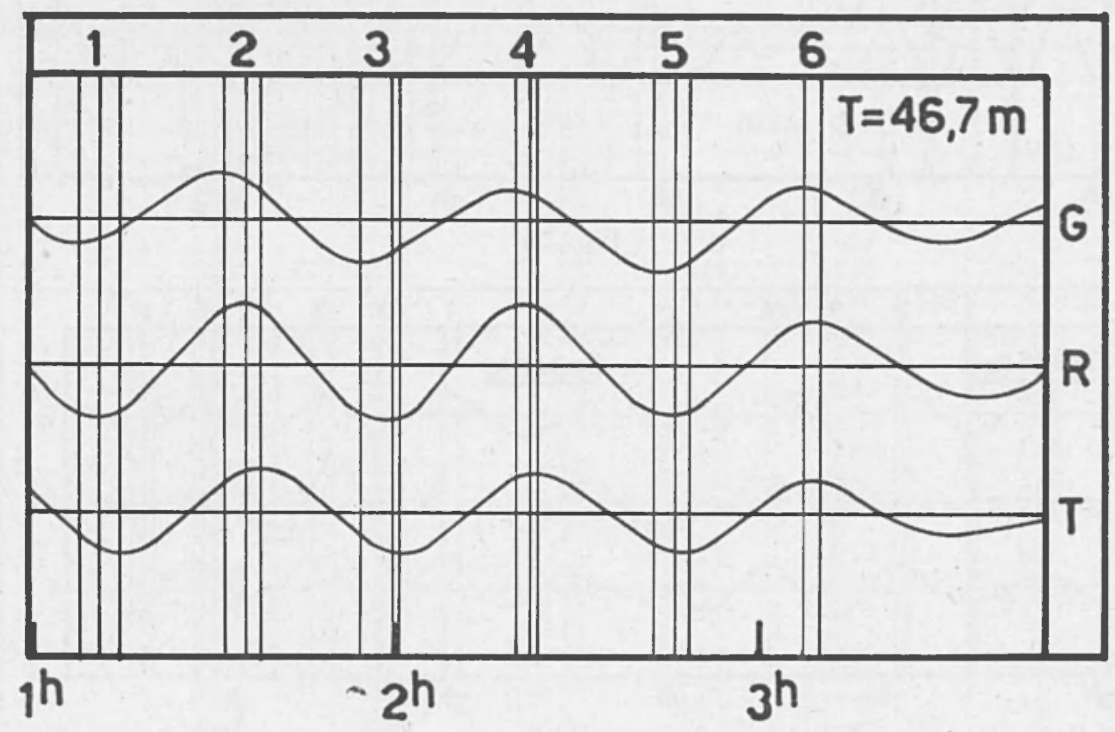

Fig. 2-b

Come si vede si tratta di gruppi di onde non eguali per periodo, succedentisi attraverso zone di sfasamento; in tutte e tre le stazioni la somiglianza delle registrazioni simultanee è evidente. L'ampiezza diversa è dovuta unicamente al fatto che l'ingrandimento non era perfettamente eguale nei tre strumenti; le costanti strumentali usate non apportano di per se stesse alcuno sfasamento apprezzalbile, sicché gli sfasamenti misurati dipendono proprio dai diversi tempi di arrivo del medesimo impulso in ognuna delle tre stazioni.

Alle differenze di fase così ottenute sulle componenti pure abbiamo applicato le formule che ci danno il coefficiente angolare $f$ della direzione di provenienza $\left({ }^{3}\right)$

e la velocità $v$

$$
f=\frac{r-p \tau}{q \tau}
$$

$$
v=\frac{q r}{\sqrt{\triangle t_{3}{ }^{2}\left(q^{2}+\psi^{2}\right)+\triangle t_{1}{ }^{2} r^{2}-2 r p \triangle t_{1} \Delta t_{2}}}
$$




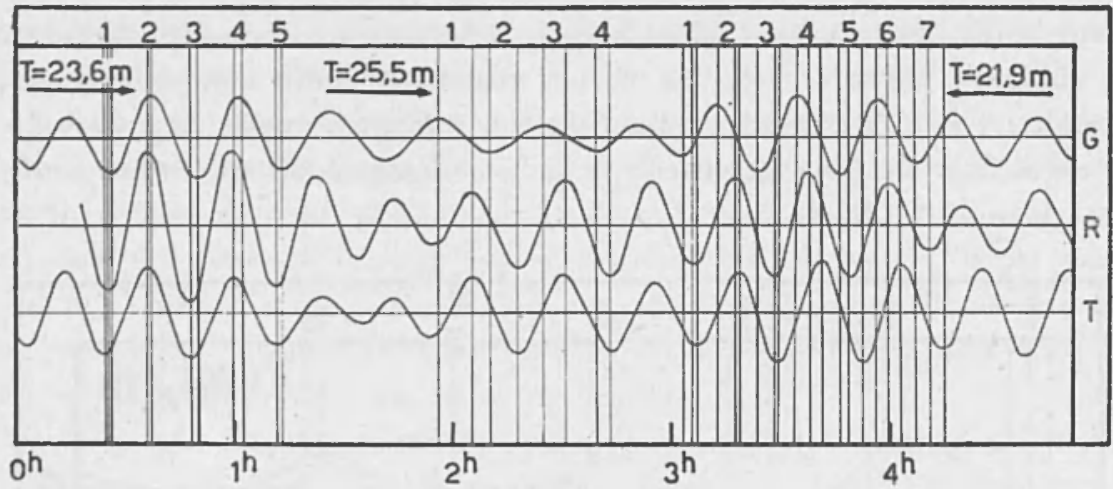

Fig. 3-a

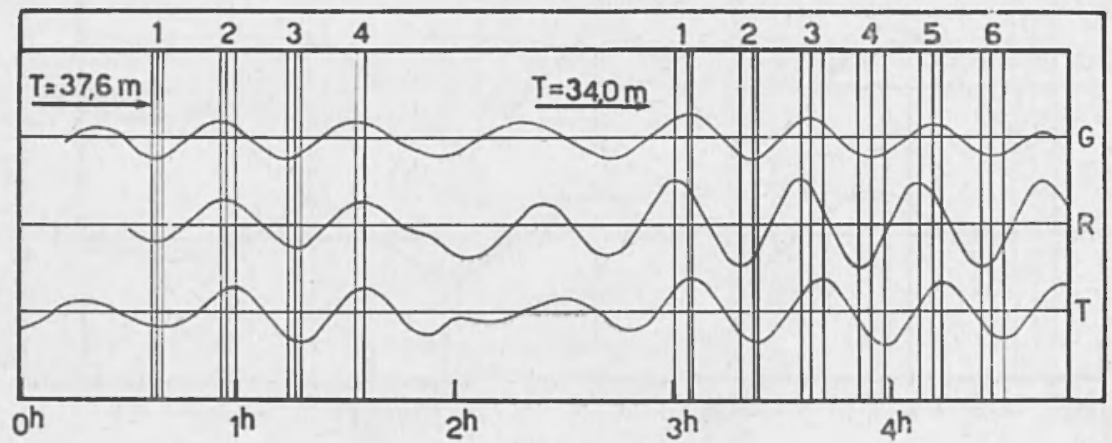

Fig. 3-b

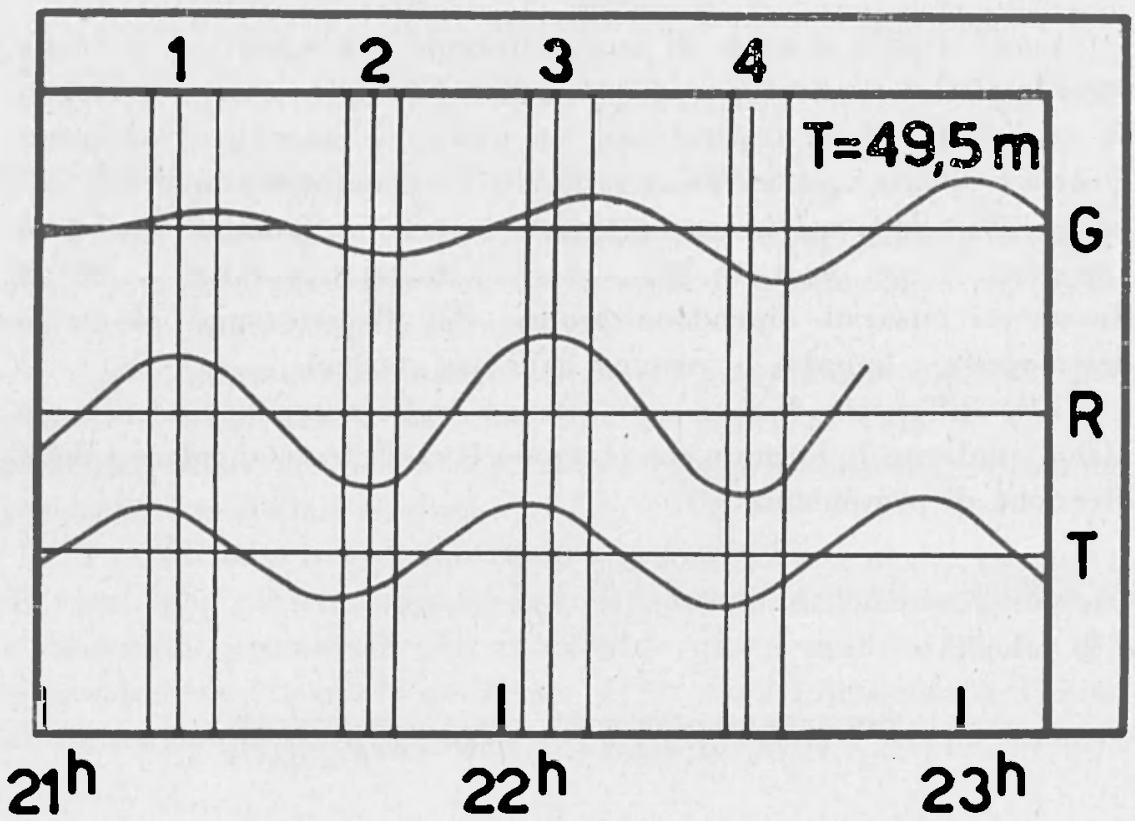

Fig, 3-c 
ove $r, p$ e $q$ dipendono dalle dimensioni e dall'orientamento del triangolo $G R T$ e valgono rispettivamente, nel nostro caso, $6450 \mathrm{~m}, 3850 \mathrm{~m}$ e $1400 \mathrm{~m}, \tau=\triangle_{\mathrm{2}} / \Delta_{1}$ con $\Delta_{2}^{\prime}=t_{\mathrm{G}}-t_{\mathrm{T}}, \triangle_{1}=t_{\mathrm{G}}-t_{\mathrm{R}}$.

Calcolando da $f$, in base all'orientamento del triangolo delle stazioni, l'azimut $a$ (in gradi contati in senso antiorario da $N$ ) e il verso, abbiamo ottenuto $\mathrm{i}$ valori riportati nella tabella seguente:

\begin{tabular}{|c|c|c|c|c|c|c|}
\hline \multirow{3}{*}{$\begin{array}{c}29-8-52 \\
0 \mathrm{~h}-5 \mathrm{~h}\end{array}$} & $\tau=\min$ & $v=m / \min$ & $\mathbf{a}$ & $\mathrm{T}=\mathrm{min}$ & $v-{ }^{m / m}$ & $\mathbf{a}$ \\
\hline & $\begin{array}{l}22,8 \\
23,6 \\
24,4 \\
23,6 \\
23,6 \\
23,6+0,3 \\
26,0 \\
26,4 \\
26,7 \\
25,2 \\
22.8 \\
25,5+0,7\end{array}$ & $\begin{array}{l}2550 \\
2460 \\
1240 \\
1240 \\
2460 \\
1990+320 \\
307 \\
411 \\
491 \\
550 \\
5: 0 \\
456+45\end{array}$ & $\begin{array}{l}+52 \\
-33 \\
-33 \\
-33 \\
-33 \\
-16+17 \\
-33 \\
-33 \\
-33 \\
-13 \\
-13 \\
-25+6\end{array}$ & $\begin{array}{l}36,6 \\
38,0 \\
37,2 \\
38,8 \\
37,6+0,5 \\
36,0 \\
34,8 \\
33,2 \\
32,8 \\
33,2 \\
3+, 0 \\
34,0 \div 0,6\end{array}$ & $\begin{array}{l}2530 \\
1160 \\
1420 \\
1240 \\
1590+316 \\
324 \\
324 \\
306 \\
275 \\
279 \\
306 \\
302+9\end{array}$ & $\begin{array}{l}+52 \\
+79 \\
+4 \\
-33 \\
+26+25 \\
+118 \\
+118 \\
+110 \\
+115 \\
+116 \\
+110 \\
+115+1\end{array}$ \\
\hline & $\begin{array}{l}22,8 \\
21,8 \\
22,0 \\
22,0 \\
21,6 \\
22,2 \\
21,2 \\
21,9+0,2\end{array}$ & $\begin{array}{l}1640 \\
775 \\
775 \\
860 \\
830 \\
860 \\
790 \\
934+120\end{array}$ & $\begin{array}{l}+\quad 4 \\
-19 \\
-19 \\
+52 \\
+71 \\
+52 \\
+33 \\
+25+14\end{array}$ & \begin{tabular}{|l|}
47,1 \\
46,8 \\
46,8 \\
47,6 \\
47,6 \\
44,4 \\
$46,7 \pm 0,4$
\end{tabular} & $\begin{array}{l}855 \\
934 \\
777 \\
1630 \\
2050 \\
1240 \\
1245+214\end{array}$ & $\begin{array}{l}+52 \\
+26 \\
-13 \\
+\quad 4 \\
-13 \\
-13 \\
+\quad 7+13\end{array}$ \\
\hline $20 \mathrm{~h}-24 \mathrm{~h}$ & $\begin{array}{l}30,6 \\
34,8 \\
35,2 \\
3,2,2 \\
35,2 \\
34,4 \\
35,2 \\
34,8 \\
36,4 \\
37,2\end{array}$ & $\begin{array}{l}526 \\
469 \\
469 \\
531 \\
312 \\
370 \\
30 \\
634 \\
790 \\
634\end{array}$ & $\begin{array}{r}+108 \\
+100 \\
+100 \\
+101 \\
+100 \\
+\quad 95 \\
+\quad 95 \\
+\quad 67 \\
+\quad 67 \\
+\quad 33\end{array}$ & $\begin{array}{l}36,4 \\
36,4 \\
37,6 \\
35,3+0,5 \\
48,0 \\
49,5 \\
50,1 \\
50,4 \\
49,5+0,6\end{array}$ & $\begin{array}{l}934 \\
855 \\
450 \\
565+54 \\
740 \\
855 \\
79 ! \\
800 \\
795+24\end{array}$ & $\begin{array}{l}+26 \\
+52 \\
+85 \\
+79+8 \\
+67 \\
+52 \\
+52 \\
+17 \\
+47+11\end{array}$ \\
\hline
\end{tabular}

Come si può notare confrontando questa tabella coi valori riportati nel lavoro $\left({ }^{3}\right)$, vi è una notevole concordanza nei valori della velocità e della direzione di provenienza dei vari impulsi rilevati direttamente dal diagramma o ottenuti mediante l'analisi periodale di questo; anche dal confronto della fig. 4 si nota la concordanza nella determinazione della direzione di provenienza; solo che $\mathrm{i}$ valori sono molto più omogenei e meno soggetti ad errore nel caso di analisi applicata, non direttamente ai diagrammi originali, ma previa separazione delle componenti. 
Avevamo detto in [3], basandoci sui poco precisi risultati di misure dirette dal microbarogramma, che non appariva alcuna netta correlazione tra velocità e periodo o direzione di provenienza, per cui concludevamo che la velocità non poteva venir considerata una caratteristica invariabile di queste onde, ma doveva venir calcolata caso per

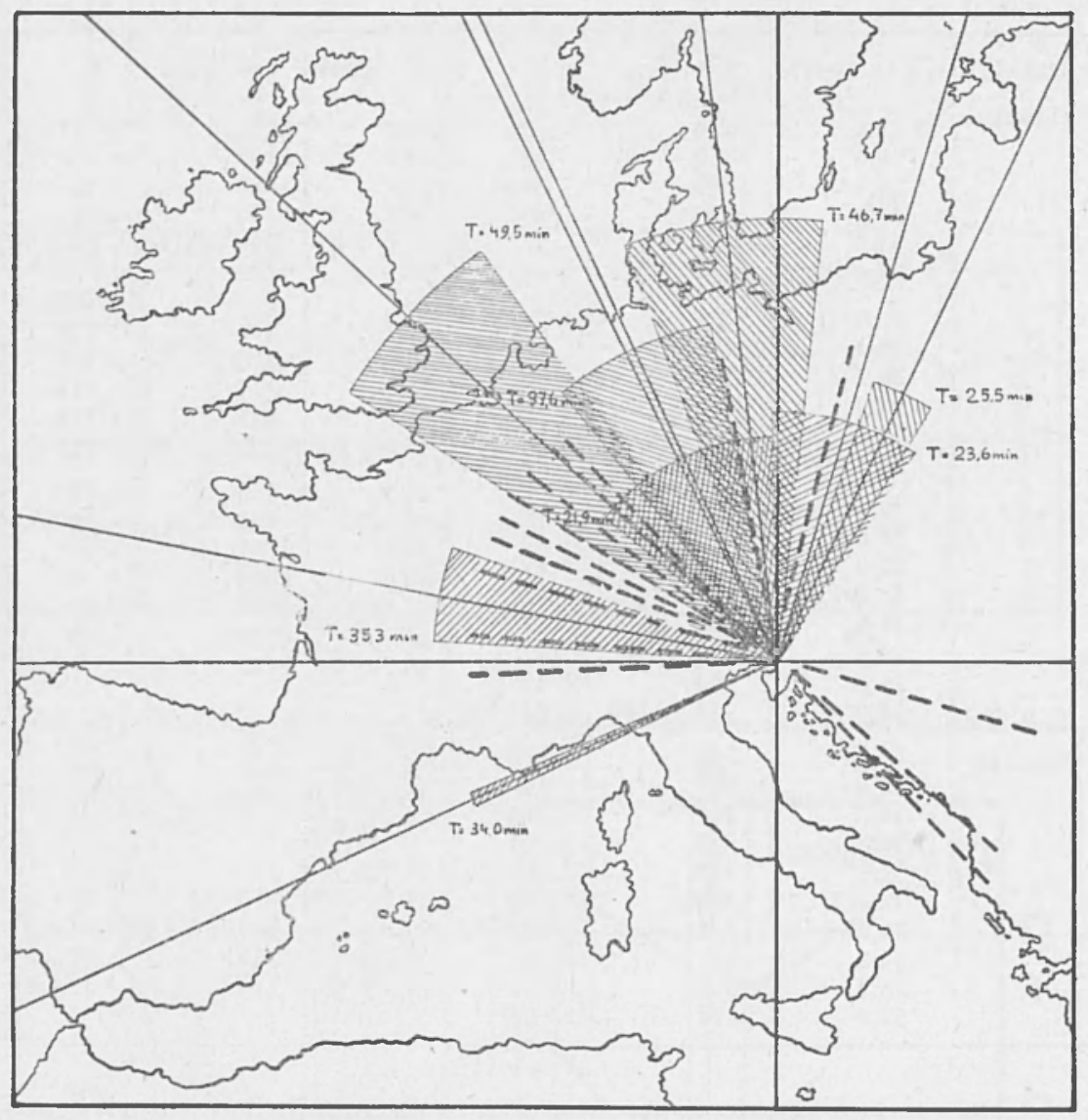

Fig. 4

caso. Con la possibilità discriminativa ben maggiore ottenuta dalla nostra scomposizione giungiamo agli stessi risultati.

Un'altra notevole miglioria che si ottiene operando sui diagrammi selezionati anziché sugli originali, riguarda la determinazione del periodo e della fase. In un diagramma originale infatti è difficile poter scegliere impulsi che, a vista, derivino dalla medesima causa e alb- 
biano perciò il medesimo periodo, come anche è difficile apprezzare il periodo stesso o misurarne lo sfasamento. Tutto questo diventa molto più facile operando sulle componenti pure ottenute dall'analisi periodale, anche se alcune di queste componenti vanno perdute per scomodità pratiche di analizzarle, e anche se errori o imprecisioni di calcolo portino, pure in questo caso, inevitabili distorsioni.

Viene inoltre dimostrata, attraverso l'analisi periodale, la continuità dei vari impulsi che formano treni d'onda abbastanza persistenti e regolari.

Queste conclusioni non possono non essere di valido aiuto per la continuazione delle ricerche con nuovi strumenti e per l'interpretazione dei loro risultati.

Istituto Nazionale di Geofisica - Osserv. Geofisico di Trieste Novembre 1954.

\section{RIASSUNTO}

Sono stati sottoposti ad analisi periodale alcuni microbarogrammi ottenuti in un gruppo di stazioni tripartite.

Con questo metodo matematico-numerico si riesce a separare le varie componenti che costituiscono $i$ grafici strumentali. Esse sono eguali per periodo e andamento dell'ampiezza in tutte e tre le stazioni del gruppo considerato; la determinazione della direzione di provenienza e della velocità applicata a queste onde semplici corrisponde, migliorandola, alla determinazione già eseguita sui diagrammi originali.

\section{SUMMARY}

We have elaborate with the periodical analisis some microbarograms obtained in a group of tripartite stations.

With this mathematicien and numerical method it is possible to separate the various components which form the instrumental graphics. They are all the same for period and development of the amplitude in all the three stations of the considerated group; the determination of the coming from direction and of the velocity applied to these simple waves is in relation, with improvement, to the determination before done on the original diagrams. 


\section{BIBLIOGRAFIA}

(1) F. Mosetri, Teoria del microbarografo Alfani, Annali di Geofisica, IV, 1951.

(2) F. Mosetri, Le onde microbariche. Rivista di Meteorologia Aeronautica, III, 1952.

(3) F. Mosettr, Primi risultati sulla localizzazione delle perturbazioni atmosferiche dall'esame delle onde microbariche, Annali di Geofisica, VI, - 4 - 1953.

(4) F. Mosertr. Principi sui filtri per le onde di pressione, Annali di Geofisica, VII, - 2 - 1954.

(5) F. MosetTI, A proposito di una questione sulle onde microbariche caratteristiche dei fronti, Annali di Geofisica, VII, 3, 1954.

(6) F. VercedlI, Analisi periodale, Tecnica Italiana, I, 1954. 